\title{
Beam Line and First Vessel Wall Shielding in HYLIFE-II
}

P.A. House

October 18, 1999

U.S. Department of Energy

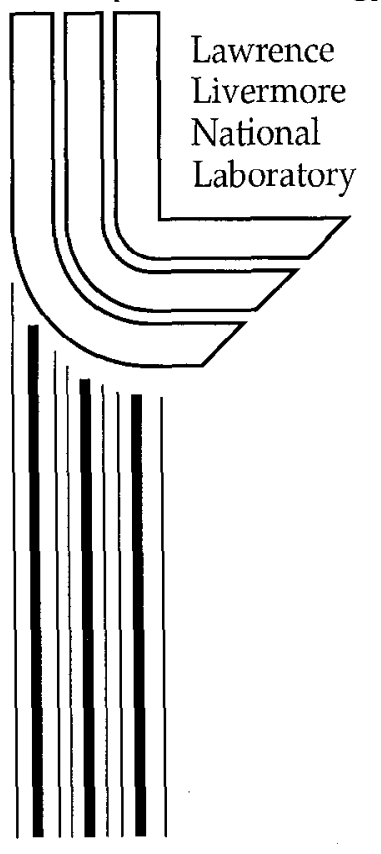




\section{DISCLAIMER}

This document was prepared as an account of work sponsored by an agency of the United States Government. Neither the United States Government nor the University of California nor any of their employees, makes any warranty, express or implied, or assumes any legal liability or responsibility for the accuracy, completeness, or usefulness of any information, apparatus, product, or process disclosed, or represents that its use would not infringe privately owned rights. Reference herein to any specific commercial product, process, or service by trade name, trademark, manufacturer, or otherwise, does not necessarily constitute or imply its endorsement, recommendation, or favoring by the United States Government or the University of California. The views and opinions of authors expressed herein do not necessarily state or reflect those of the United States Government or the University of California, and shall not be used for advertising or product endorsement purposes.

Work performed under the auspices of the U.S. Department of Energy by the University of California Lawrence Livermore National Laboratory under Contract W-7405-Eng-48.

This report has been reproduced

directly from the best available copy.

Available to DOE and DOE contractors from the

Office of Scientific and Technical Information

P.O. Box 62, Oak Ridge, TN 37831

Prices available from (423) 576-8401

http://apollo.osti.gov/bridge/

Available to the public from the

National Technical Information Service

U.S. Department of Commerce

5285 Port Royal Rd.,

Springfield, VA 22161

http://www.ntis.gov/

OR

Lawrence Livermore National Laboratory

Technical Information Department's Digital Library

http://www.llnl.gov/tid/Library.html 


\title{
Beam Line and First Vessel Wall Shielding in HYLIFE-II*
}

\author{
Palmer A. House \\ University of California \\ Lawrence Livermore National Laboratory \\ P.O. Box 808 \\ Livermore, California 94550 \\ October 18, 1999
}

\begin{abstract}
The present Heavy Ion Driver design for HYLIFE-II requires 96 beams from each side, or a total of 192 beams. The beams are separated from each other, at present, by an angle of 4.25 degrees. Two sets of Flibe (molten Salt) jets, 90 degrees apart, are used for x-ray and neutron shielding of the first vessel wall. Space between jets result in no shielding of each beam tube and a surrounding rectangular area of vessel wall. A vortex shielding device is proposed to provide this additional required shielding. This report describes the near ideal quality of Flibe jets that are needed, for the shielding design to be practical. First wall shielding at locations other than close proximity to the beam lines, is accomplished by oscillating flow and extensions to the horizontal beam protection jets.
\end{abstract}

\section{Introduction}

HYLIFE-II is a design study of a $1 \mathrm{GW}_{\mathrm{e}}$ inertial fusion energy power plant (Moir, 1994). Shielding of the metal parts of the structure is accomplished by a "liquid wall" using Molten Flibe $\left(\mathrm{Li}_{2} \mathrm{BeF}_{4}\right)$. The heavy ion driver has evolved from 12 beams from one side (Moir, 1994) to the present 96 beams per side. The difficulty in providing adequate beam line shielding increases as the space between beams decreases.

Figure 1 illustrates the beam line shielding jets used in conjunction with the oscillating flow debris clearing system. Jets pass between the beam lines, but require some clearance to insure no attenuation of the beams. In Fig.1 the first set of jets is horizontal while the next set is vertical. All beams converge on the target (working point) at the center of the vessel. Inside the reactor vessel each heavy ion beam is conical with a half angle of 0.415 degrees (Lee, 1999). The 4.25 degree angle between beams evolved from beam size 
information and focus magnet locations (Lee, 1999). For a 30 year plant lifetime a minimum thickness of $0.5 \mathrm{~m}$ of Flibe must be present on any path to a vessel part, from the working point (WP). A view from the working point toward the vessel wall will leave the beam tube and a square or rectangular zone surrounding the beam unshielded. A vortex shielding device (Fig. 2) can provide this needed shielding, if the cross flow (horizontal and vertical) shielding jets can have a small (about $1 \mathrm{~cm}$ ), consistent spacing from the beams. Larger space between the jets and beams, at any location, will jeopardize the cross flow / vortex device shielding method.

\section{Shielding Patterns}

Figure 3 shows the shielding projection for 16 of the 96 beams at a $3 \mathrm{~m}$ radius from the WP. A space (clearance plus ripple) of $0.9 \mathrm{~cm}$ between the beams and jets is assigned for the horizontal jets and a $1.4 \mathrm{~cm}$ space for the vertical jets. Curvature of the horizontal jets from gravity is not shown. Hopefully beam positions can be changed to accommodate this curvature by beam steering. The curvature can be reduced by increasing the jet velocity, unless the higher velocity causes additional complications (such as jet stability and spreading). The spaces of Fig. 3 are near the maximum that can be tolerated in order to have the room needed for the vortex shielding device. In Fig. 4 the beam to jet spaces of Fig. 3 have been doubled. Spaces are now $1.8 \mathrm{~cm}$ from beam to horizontal jet and 2.8 $\mathrm{cm}$ from jet to vertical jet. There is no longer shielded room available for the vortex device hardware.

\section{Vortex Shielding Device}

The purpose of the apparatus is to provide a flowing hollow cylinder of Flibe for shielding. The liquid must spin in order to maintain the hollow core. The vortex shielding device (House, Moir, 1999) shown in Fig. 2 is configured to fit in the very limited room available with the 96 beam array. Flibe under pressure enters an annular duct and flows toward the WP where it is turned $180^{\circ}$ and enters the turning blades. Flibe exiting the turning blades has both spin (whirl) and axial velocities. Mechanical spoilers are used to reduce these velocities, in order to increase the flow cross sectional area. This is necessary in order to hide the metal device inside tip behind the shaded area of Fig. 3. As shown in Fig. 2 the thickened whirling liquid also shields the beam tube. The apparatus would be constructed from metal (probably 304 stainless steel). The apparatus is stationary, only the molten salt is moving. 


\section{Jet Quality Problems}

Dimensional stability and accuracy in placement of the jets, in the tightly packed 96 beam array, is paramount. The maximum beam to jet space that can be allowed, for any beam, are $0.9 \mathrm{~cm}$ for horizontal jets and $1.4 \mathrm{~cm}$ for the vertical jets. The force of gravity is one of the problems. It causes curvature in the horizontal jets and area or density reduction in the vertical jets. As mentioned above beam steering can potentially compensate for the jet curvature. If the gravity induced increase in velocity of the vertical jets result in a density reduction rather than a dimensional reduction, then wider (than 0.5 m) jets will compensate. If dimensional reduction occurs as a result of the acceleration of gravity, then higher start velocities will partially compensate. Fortunately the effects of gravity are predictable and do not appear to be too serious.

A more serious problem of jet stability is "ripple". Shear forces, caused by stationery nozzle walls and moving liquid, induce turbulence in the surface layer of liquid. Once the jet leaves the nozzle surface, roughness increases with distance traveled. Surface waves could easily be larger than the small clearance available, and as a result interfere with the beams. Jet stability for single nozzles can be improved by upstream flow straighteners and by scraping off the surface turbulent layer of liquid leaving the nozzle. Both of these techniques may not be practical for our thin closely spaced jets. For example the center horizontal jet size (Fig. 1) is $4.77 \times 7.75 \times 50 \mathrm{~cm}$. The center vertical jet size is $7.35 \mathrm{x}$ 10.33 x $50 \mathrm{~cm}$. Minimum space (jet to jet) is $3.39 \mathrm{~cm}$ for horizontal jets and $5.26 \mathrm{~cm}$ for the vertical jets. The maximum jet and nozzle size is shown in Fig. 5 for the vertical jets at the $2.2 \mathrm{~m}$ location.

\section{Ripple magnitude that can be tolerated}

Space between the jets and beams is needed for both the ripple build up and lack of perfect aiming the jets. With so little space between beams the accuracy of aiming needed might not be attainable. Minor velocity variation (caused by pressure changes) in the vertical jets is not a factor but it results in a different trajectory for the horizontal jets, due to the accelcration of gravity. Another factor cffecting ripple and aiming is the placement of the nozzles. In Fig. 6 it can be seen that the vertical nozzles are placed close to the beam paths, since shielding of these nozzles is accomplished by an extension of the horizontal flow. The horizontal nozzles must be placed further away $(1.15 \mathrm{~m})$ from the beams in order to have $0.5 \mathrm{~m}$ minimum of shielding from the oscillating flow Flibe, see Fig. 7. The problems of ripple growth and aiming accuracy are both aggravated as the 
distance between nozzles and the beams is increased. In this report it is assumed that the magnitude of surface ripple (waves) increases linearly with distance from the nozzle exit. This linear variation needs to be verified by experiment. The magnitude of ripple is the distance from wave peak to wave valley.

At the $2.2 \mathrm{~m}$ plane, Fig. 8 , the parameters used in the calculation of maximum allowable vertical jet ripple are shown. Distance from the vertical nozzle to the beam array centerline is $0.85 \mathrm{~m}$. After another $0.76 \mathrm{~m}$ of travel the jets reach the end of the beam array, thus the total jet length is $0.85+0.76=1.61 \mathrm{~m}$. If we allow $0.5 \mathrm{~cm}$ of clearance for aiming, then $1.4-0.5=0.9 \mathrm{~cm}$ is available for ripple. If " $\mathrm{a}$ " is the magnitude of ripple, then: $\mathrm{a}=0.009 / 1.61=0.0056 \mathrm{~m} / \mathrm{m}$ for the vertical jets. At the $1.6 \mathrm{~m}$ plane, Fig. 9 , the parameters used in the calculation of maximum allowable horizontal jet ripple are shown. Distance from the horizontal nozzle to the beam array centerline is $1.7 \mathrm{~m}$. After another $0.56 \mathrm{~m}$ of travel the jets reach the end of the beam array, thus the total jet length is $1.7+$ $0.56=2.26 \mathrm{~m}$. If we again allow $0.5 \mathrm{~cm}$ of clearance for aiming, then $0.9-0.5=0.4 \mathrm{~cm}$ is available for ripple The magnitude of ripple, is then: $a=0.004 / 2.26=0.0018 \mathrm{~m} / \mathrm{m}$ for the horizontal jets.

Allowance for aiming accuracy for vertical jets is only, arctangent $0.005 / 1.61=0.18$ degrees. The allowance for aiming accuracy for horizontal jets is only arctangent $0.005 / 2.26=0.13$ degrees. It may be difficult in practice it attain this accuracy of aiming for fixed nozzles that are not individually adjustable. For example, if the nozzles were configured as shown in Fig. 5, then the end nozzles might have a slightly different flow direction, due to the influence of the end walls, compared to the other 9 nozzles.

\section{Conclusions}

The parameters needed to achieve satisfactory first wall and beam tube shielding, in the beam entry zone, have been calculated. The 192 beam system ( 96 beams per side) in its present form of 4.25 degrees between beams may not have sufficient room for adequate shielding to be realistically practical. It is likely that changes will need to be made to attain more space between beams. In order to know how much space is required, experimental results of the magnitude of both the ripple factor " $a$ " and attainable nozzlc aiming accuracy are needed for closely spaced parallel nozzles.

This report has addressed the shielding requirements at shot time. Energy deposition in the shielding material (Flibe), at the time of detonation, will drive the cross flow streams into each other and create a chaotic flow condition. A complete recovery to a jet steady 
flow condition is required in the fraction of a second available between shots. Once again, experimental results on recovery time are required for power reactor design to progress.

* Work performed under the auspice of the U. S. Department of Energy by Lawrence Livermore Laboratory under Contract W-7405-ENG-48.

\section{References}

1 R. W. Moir, et al (1994), "HYLIFE-II: A Molten Salt Inertial Fusion Energy Power Plant Design-Final Report”, Fusion Technology 25, 5-25 (1994)

2. E. P. Lee, Lawrence Berkeley National Laboratory, private communication, 6/15/99.

3. P. A. House, R. W. Moir (1999), "Beam Line Protection Vortex", Patent Disclosure, IL-10506 
Cross Flow Shielding Jets (used with the oscillating center flow system)

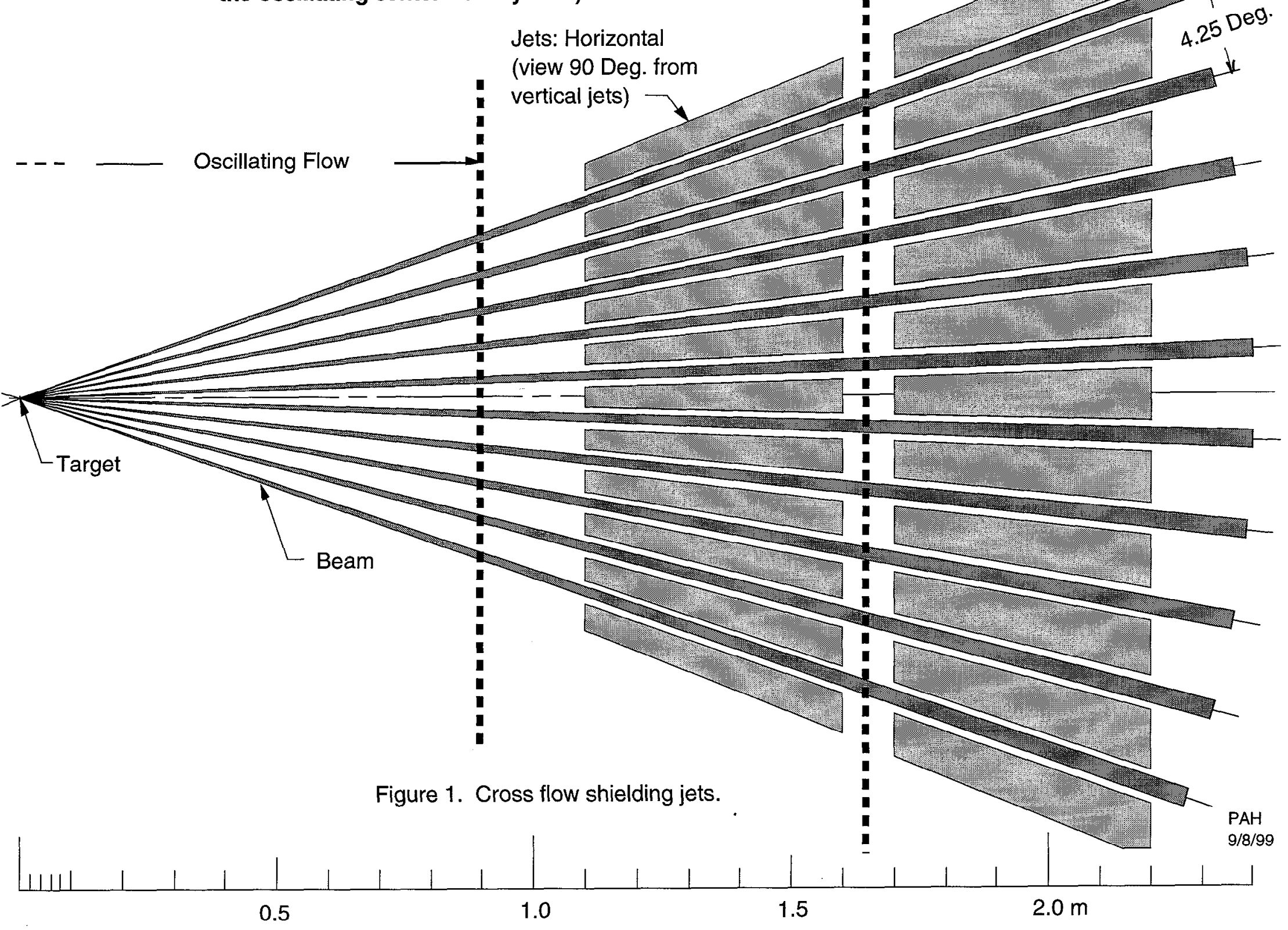




\section{Vortex Shielding Device, Design Concept}

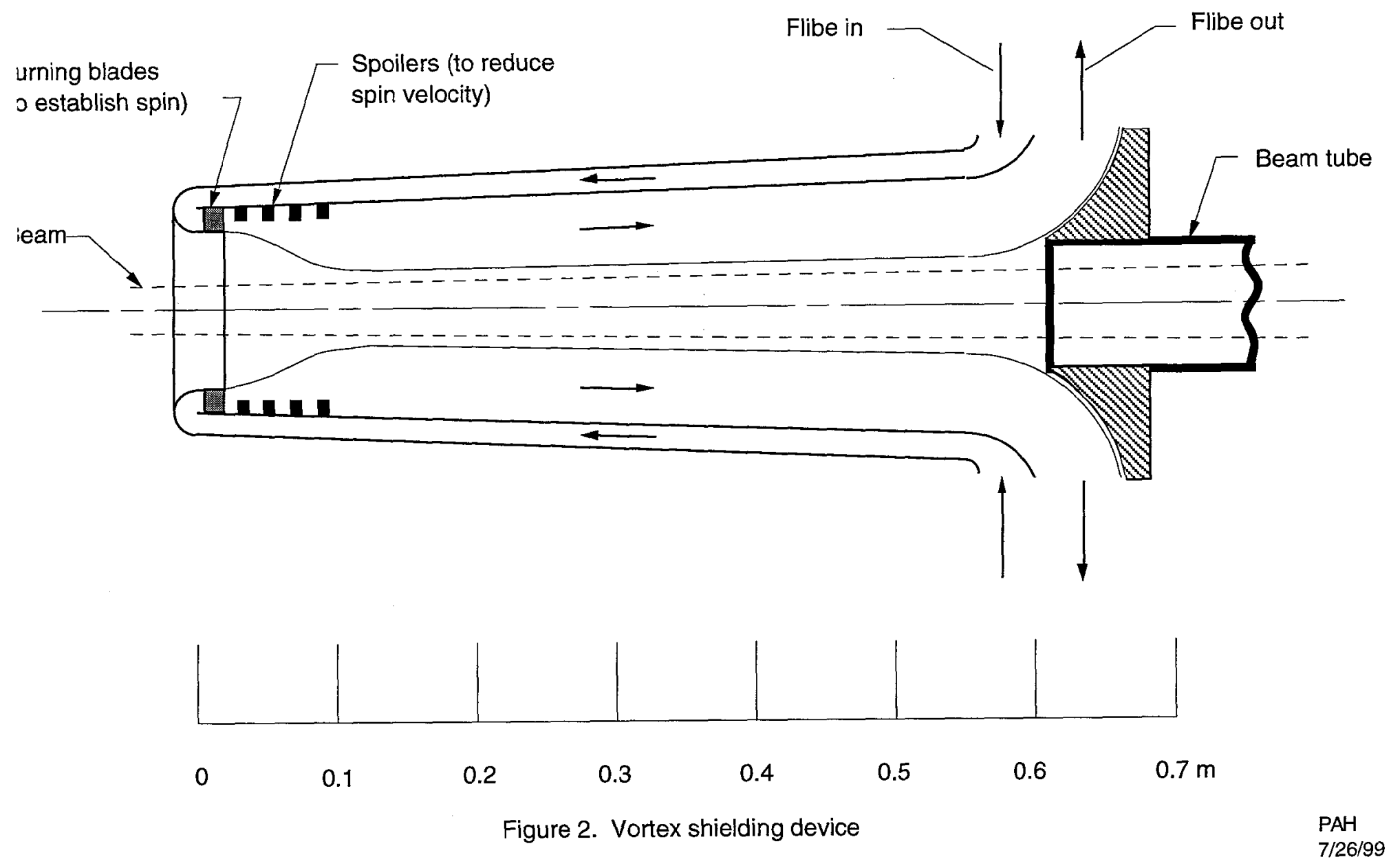


Shielding Jet Pattern at $3 \mathrm{~m}$ (Curvature from gravity not shown)

- Oscillating flow system (center 16 of 92 beams shown)

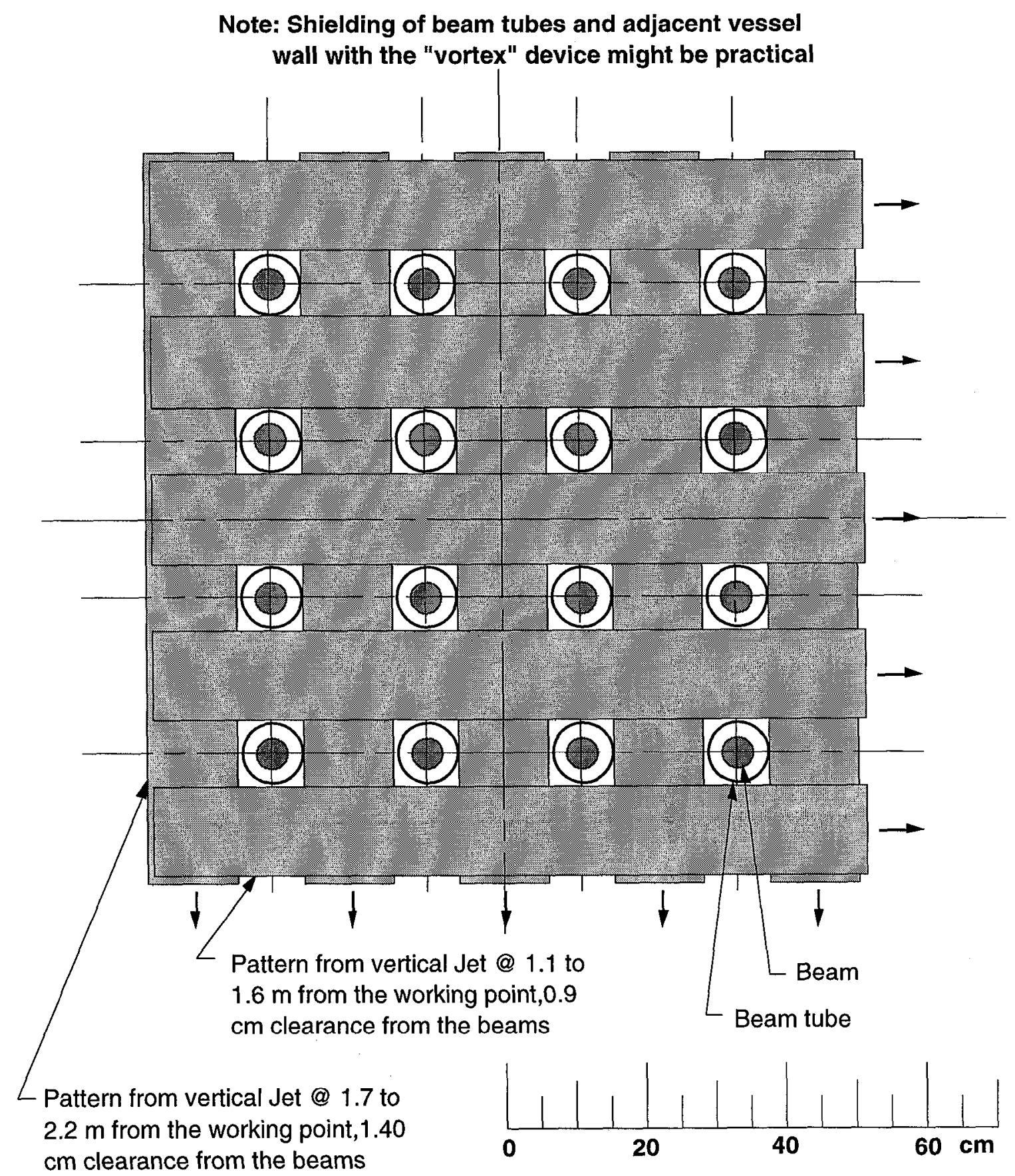

Fig. 3. Shielding pattern from the largest jet to beam clearance that can be tolerated 
Shielding Jet Pattern at $3 \mathrm{~m}$ (Curvature from gravity not shown)

- Oscillating flow system (center 16 of 92 beams shown)

Note: Shielding of beam tubes and adjacent vessel wall with the "vortex" device is no longer feasible

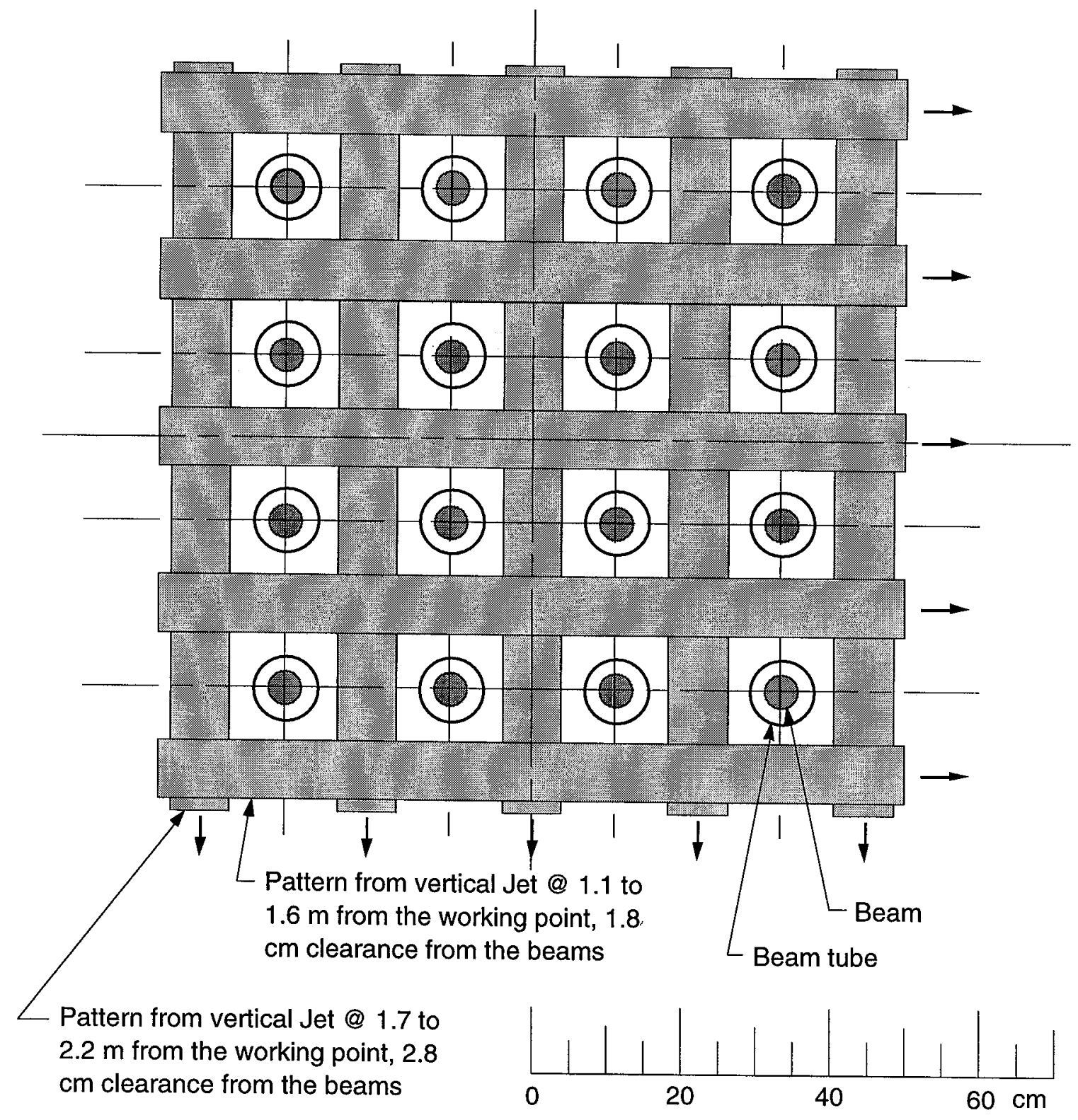

Fig. 4. Jet to beam clearances doubled from the largest that can be tolerated. 


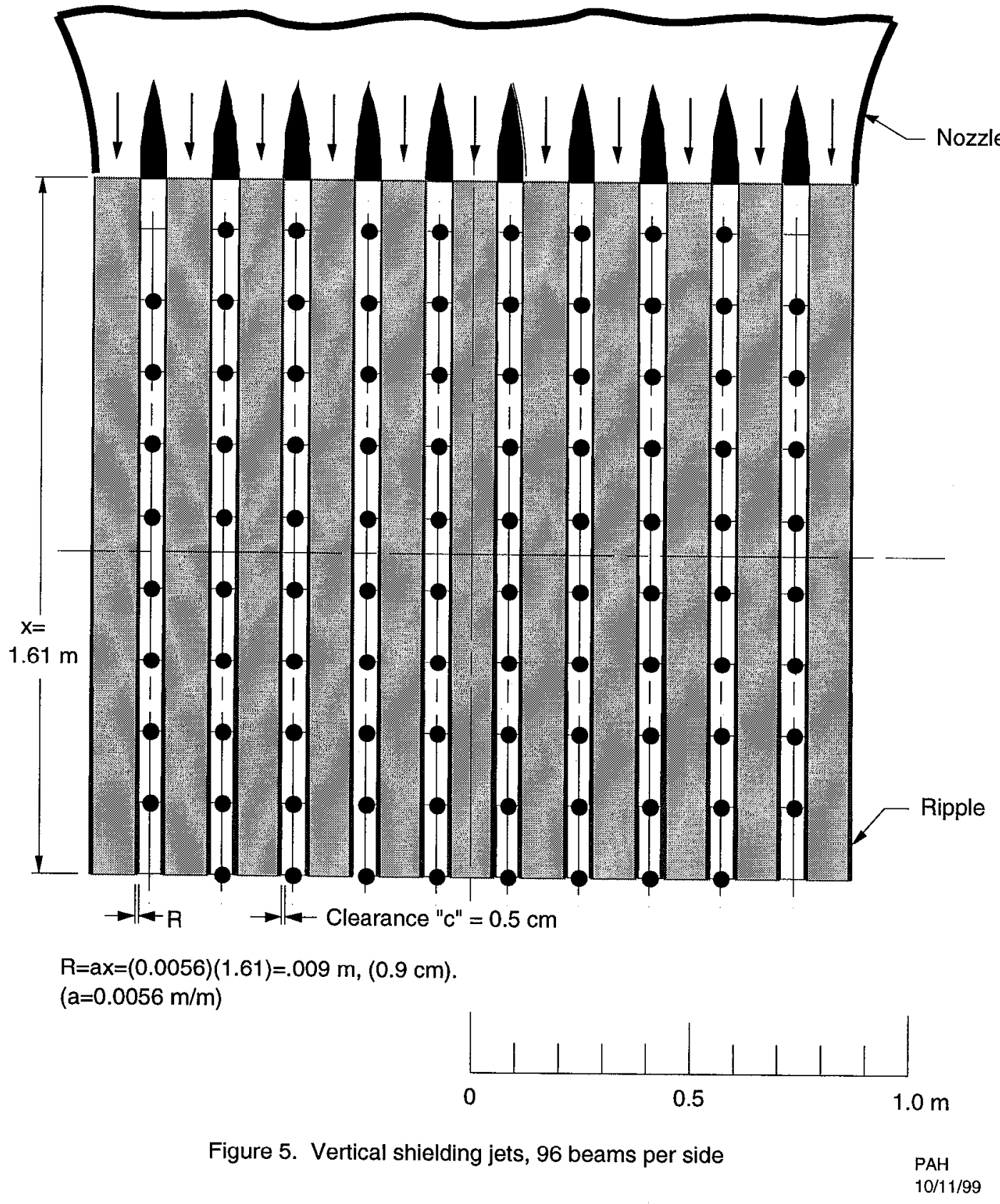




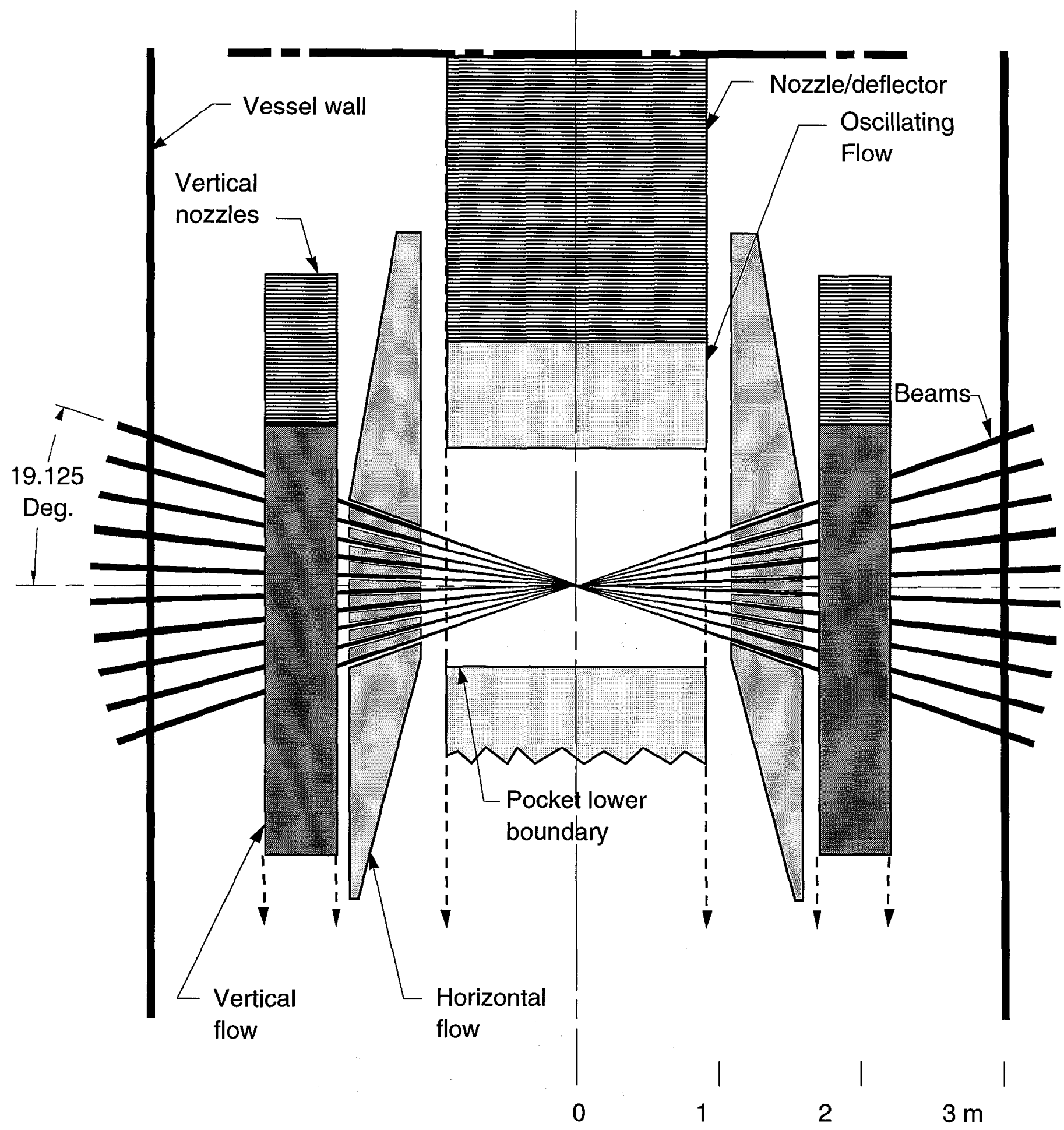

Figure 6. Flibe shielding jets, side view 
HYLIFE II With 96 beams per side

(Plan View)

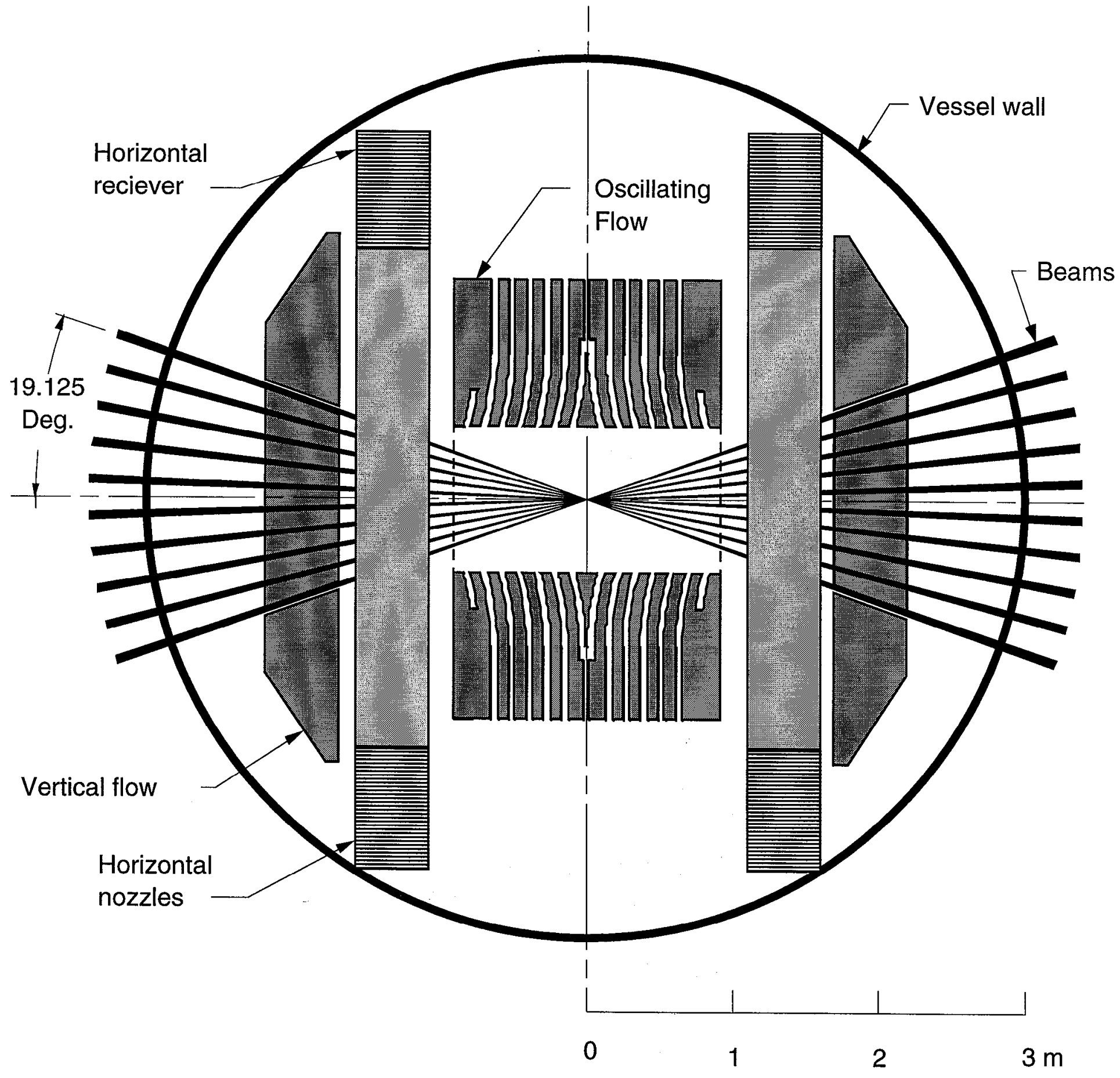

Figure 7. Flibe shielding jets, plan view 


\section{Vertical Jet Ripple}

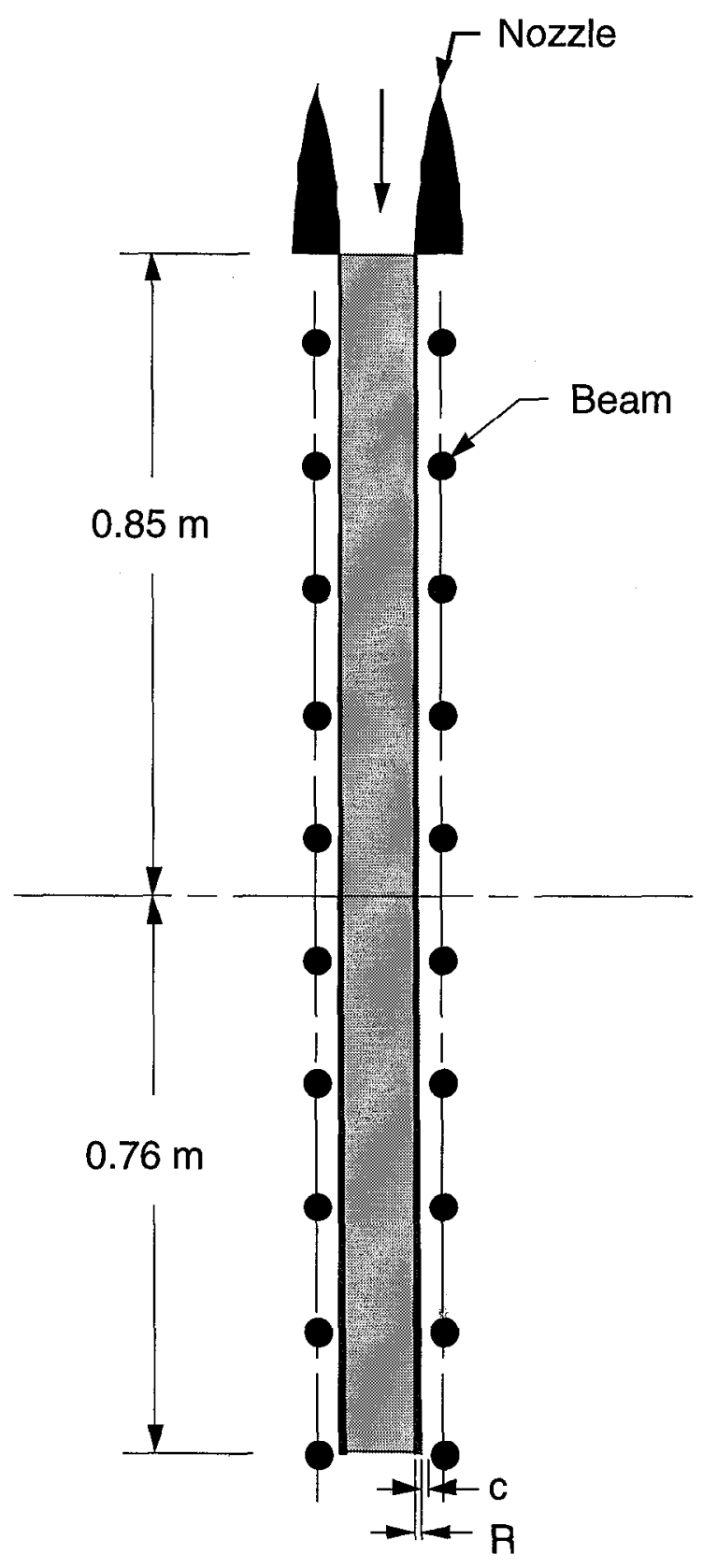

Calculation:

$X=$ length of jet, $m$

$\mathrm{R}=$ magnitude of ripple, $\mathrm{m}$

$c=$ clearance for aiming $=0.005 \mathrm{~m}$

$x=0.85+0.76=1.61 \mathrm{~m}$

$\mathrm{R}+\mathrm{c}=1.4 \mathrm{~cm}=0.014 \mathrm{~m}$

$R=0.014-0.005=0.009 \mathrm{~m}$.

$\mathrm{a}=$ ripple factor, $\mathrm{m} / \mathrm{m}$

$\mathrm{a}=\mathrm{R} / \mathrm{x}=0.009 / 1.61=0.0056 \mathrm{~m} / \mathrm{m}$

Figure 8. Vertical jet, ripple factor 


\section{Horlzontal Jet Ripple}

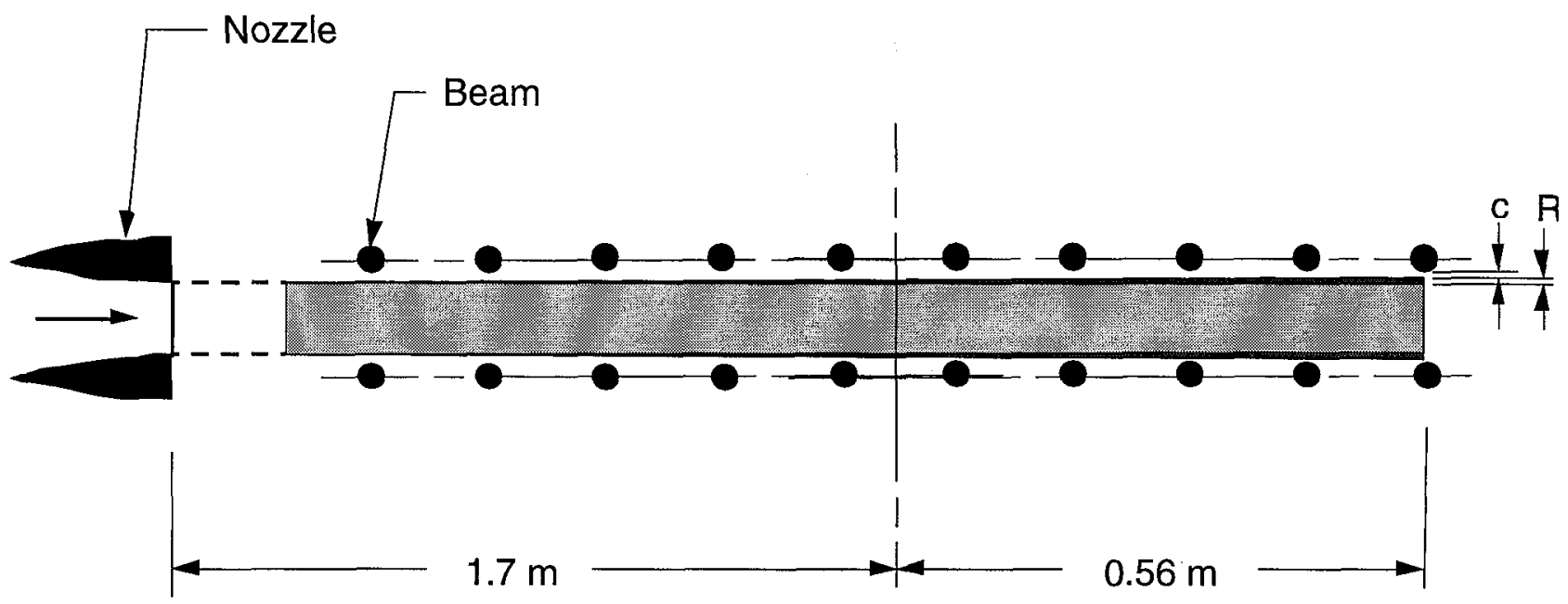

Calculation:

$X=$ length of jet, $m$

$\mathrm{R}=$ magnitude of ripple, $\mathrm{m}$

$c=$ clearance for aiming $=0.005 \mathrm{~m}$

$\mathrm{a}=$ ripple factor, $\mathrm{m} / \mathrm{m}$ $x=1.7+0.56=2.26 m$

$\mathrm{R}+\mathrm{c}=0.9 \mathrm{~cm}=0.009 \mathrm{~m}$

$R=0.009-0.005=0.004 \mathrm{~m}$.

$a=R / x=0.004 / 2.26=0.0018 \mathrm{~m} / \mathrm{m}$

Figure 9. Horizontal jet, ripple factor 